\title{
Redirecting $N$-acetylaspartate metabolism in the central nervous system normalizes myelination and rescues Canavan disease
}

\author{
Dominic J. Gessler, ${ }^{1,2,3,4}$ Danning Li, ${ }^{2}$ Hongxia Xu, ${ }^{2,5}$ Qin Su, ${ }^{2}$ Julio Sanmiguel, ${ }^{2}$ Serafettin Tuncer, ${ }^{6}$ \\ Constance Moore, ${ }^{7}$ Jean King, ${ }^{7}$ Reuben Matalon, ${ }^{8}$ and Guangping Gao $0^{1,2,9}$ \\ 'Department of Microbiology and Physiological Systems, ${ }^{2}$ Horae Gene Therapy Center, University of Massachusetts, \\ Worcester, Massachusetts, USA. ${ }^{3}$ University Hospital Heidelberg, Centre for Child and Adolescent Medicine, Division of \\ Child Neurology and Metabolic Medicine, ${ }^{4}$ Ruprecht-Karls University, Medical School, Heidelberg, Germany. ${ }^{5}$ University \\ of Science and Technology of Kunming, China. ${ }^{6} \mathrm{Heinrich}$ Heine University, Medical School, Duesseldorf, Germany. ${ }^{7}$ Center \\ for Comparative Neuroimaging, Department of Psychiatry, University of Massachusetts Medical School, Worcester, \\ Massachusetts, USA. ${ }^{8}$ University of Texas Medical Branch, Galveston, USA. ${ }^{9}$ State Key Laboratory of Biotherapy, West \\ China Hospital, Sichuan University, Chengdu, China.
}

Canavan disease (CD) is a debilitating and lethal leukodystrophy caused by mutations in the aspartoacylase (ASPA) gene and the resulting defect in $\mathrm{N}$-acetylaspartate (NAA) metabolism in the CNS and peripheral tissues. Recombinant adeno-associated virus (rAAV) has the ability to cross the blood-brain barrier and widely transduce the CNS. We developed a rAAV-based and optimized gene replacement therapy, which achieves early, complete, and sustained rescue of the lethal disease phenotype in CD mice. Our treatment results in a super-mouse phenotype, increasing motor performance of treated CD mice beyond that of WT control mice. We demonstrate that this rescue is oligodendrocyte independent, and that gene correction in astrocytes is sufficient, suggesting that the establishment of an astrocyte-based alternative metabolic sink for NAA is a key mechanism for efficacious disease rescue and the super-mouse phenotype. Importantly, the use of clinically translatable high-field imaging tools enables the noninvasive monitoring and prediction of therapeutic outcomes for $C D$ and might enable further investigation of NAA-related cognitive function.

Conflict of interest: G. Gao is a co-founder of Voyager Therapeutics, a biopharmaceutical company, and holds equity in the company. C. Gao and D.J. Cessler are inventors on a patent pending (PCT/US2016/058197) that is relevant to the content of this manuscript, which may result in potential royalties if granted and licensed.

Submitted: September 20, 2016 Accepted: December 21, 2016 Published: February 9, 2017

Reference information: JCI Insight. 2017;2(3):e90807. https:// doi.org/10.1172/jci.insight.90807.

\section{Introduction}

$N$-acetylaspartate (NAA) is one of the most abundant molecules in the mammalian CNS $(1,2)$. It is synthesized in neurons and hydrolyzed by oligodendroglial aspartoacylase (ASPA). Dysfunction or deficiency of ASPA causes NAA accumulation in the CNS and urine (aciduria) (3-8), and leads to Canavan disease (CD), a lethal pediatric leukodystrophy. Heterozygous carriers of mutations in ASPA are asymptomatic, but children who are homozygous for the mutation show reduced head control, lack of visual fixation, and muscular hypotonia within the first weeks or months of life. Later, children develop spasticity, ataxia, and seizure. Most CD patients die within the first years of life, although some live to adolescence or adulthood (9-12). Neuropathology reveals the formation of vacuoles and loss of myelin structures throughout the CNS, particularly in deep brain regions such as the thalamus, brain stem, and cerebellum $(13,14)$. A diagnostic hallmark on magnetic resonance spectrometry (MRS) is the accumulation of NAA and on MRI the loss of white matter and accumulation of water, suggestive of brain edema. Besides diagnostic applications, newer MRI-based modalities are promising to monitor disease progression and therapeutic response. For example, diffusion tensor imaging (DTI) detects directional water movements to evaluate the integrity of neuronal fiber tracts, which can be quantified as fractional anisotropy (FA) values (15). This information is particularly useful in leukodystrophies such as $\mathrm{CD}$ where extensive loss of white matter and its associated neuronal tracts occurs. However, it is limited to detecting neuroanatomical changes and does not provide any functional evaluation of the brain. In contrast, functional MRI (fMRI) allows the recording of functional connectivity between brain regions by measuring the blood oxygen level-dependent signal (16). The sensitivity of fMRI in clinical settings 
allows the detection of functional changes in the CNS after traumatic brain injury where conventional MRI is incapable of showing any pathology (17-19). In preclinical rodent models, fMRI is primarily used to study brain function in a neuroscience setting (20).

Although Myrtelle Canavan described the clinical symptoms of CD for the first time in 1931 (21), the genetic cause was unknown until Matalon et al. discovered the defect in ASPA over 50 years later (7). This discovery led to the cloning of the human ASPA gene in 1993, which was a milestone towards genetic diagnosis, gene therapy, and understanding the function of NAA (6). CD became the first CNS disorder to be treated using recombinant adeno-associated virus (rAAV) for human gene therapy in an early trial demonstrating long-term safety for rAAV-mediated gene delivery of human ASPA (hASPA) to the CNS (22). Around that time, Matalon et al. published the first ASPA knockout mouse model for CD (CD KO mouse), paving the way for studies of pathophysiology and preclinical gene therapy (23). Although $A S P A$ mutations are responsible for $\mathrm{CD}$, the physiologic and pathomechanistic roles of NAA are still unknown. Current hypotheses for the homeostasis of NAA in the CNS cover a wide spectrum of functions, including molecular water pump (24), acetate donor for myelin lipids (25), histone acetylation (26), and neuronal differentiation (27). Understanding how NAA metabolism is coordinated in neurons and glial cells has been another major challenge.

Therapeutically, non-gene therapy strategies to reduce NAA or to recover myelination have failed to show substantial improvement in animal models or in patients and remain experimental (28-30). Preclinical gene therapy with rAAV2 and intracranial delivery strategies have also failed to effectively rescue the disease phenotype $(31,32)$. In contrast, we have shown that a single i.v. injection of rAAV9 ubiquitously expressing hASPA as our 1st generation CD gene therapy restores the overall survival and achieves partial but unsustained rescue of the severe neurologic phenotypes in CD KO mice (33). However, the propensity of some CNS-tropic rAAVs to predominantly transduce neurons and astrocytes $(34,35)$ raised doubts regarding the usefulness of this approach for $\mathrm{CD}$, which is considered an oligodendrocyte-related disorder.

In the current study, we demonstrate the complete rescue of $\mathrm{CD}$ in a preclinical mouse model by rAAVhASPA gene replacement therapy and its evaluation and potential outcome prediction by high-field DTI and fMRI imaging. We show that the potency of our $h A S P A$ expression system allows further investigation of NAA metabolism. Finally, our results demonstrate that direct treatment of oligodendrocytes is not required for therapeutic success and astrocytes play an important role in NAA metabolism.

\section{Results}

Transgene cassette optimization achieves a rapid therapeutic response in CD KO mice. To overcome the challenge of efficacy and sustainability of our first-generation gene therapy (33), we chose to increase hASPA expression from the vector genome without changing the parameters of administration route, vector dose, or serotype. Comparing the effect of Kozak sequence and cDNA optimization, we designed 2 new expression cassettes with either a half or full Kozak sequence and a codon-optimized cDNA and named them 2nd and 3rd generation vectors (Figure 1A; hereafter referred to as 2 nd or 3 rd generation). In order to test the translatability in vivo, all expression cassettes were packaged into the highly CNS-tropic rAAV9 vector for i.v. gene delivery to the CNS. Untreated and 1st generation-treated mice displayed the characteristic low weight (33) at around P14 to P16 (Supplemental Figure 1; supplemental material available online with this article; https://doi. org/10.1172/jci.insight.90807DS1). However, 2nd and 3rd generation-treated mice ( $n=10$ each) paralleled WT weight gain, suggesting a more rapid onset of therapeutic transgene expression levels (Supplemental Figure 1). To evaluate neuropathology in the living mice, MRI was performed at P42 for treated and P28 for untreated animals. While untreated and 1st generation-treated mice showed strong hyperintense signals (white signals) on T2 sequence, particularly in deeper brain regions, the signals appeared isotense (unremarkable) in the 2nd and 3rd generation-treated groups (Supplemental Figure 2), suggesting normalization of brain edema. This therapeutic effect was corroborated by normalized NAA levels in the 2nd and 3rd generation treatment groups on MRS, which also coincided with ASPA protein expression levels (Figure 1, B and C). Finally, mice were assessed for neurohistopathology at P25. Again, the 1st generation-treated mice still showed vacuolization of the CNS, although to a lesser extent than untreated mice. In contrast, 2nd and 3rd generation-treated mouse brains were indistinguishable from WT, demonstrating that the improved gene therapy effectively mitigates neuropathology (Supplemental Figure 3 and data not shown).

Third generation-treated CD KO mice sustainably outperform control animals. CD KO mice recapitulate the clinical phenotype of $\mathrm{CD}$ patients, presenting with ataxia, disbalance, muscle weakness, and cognitive 


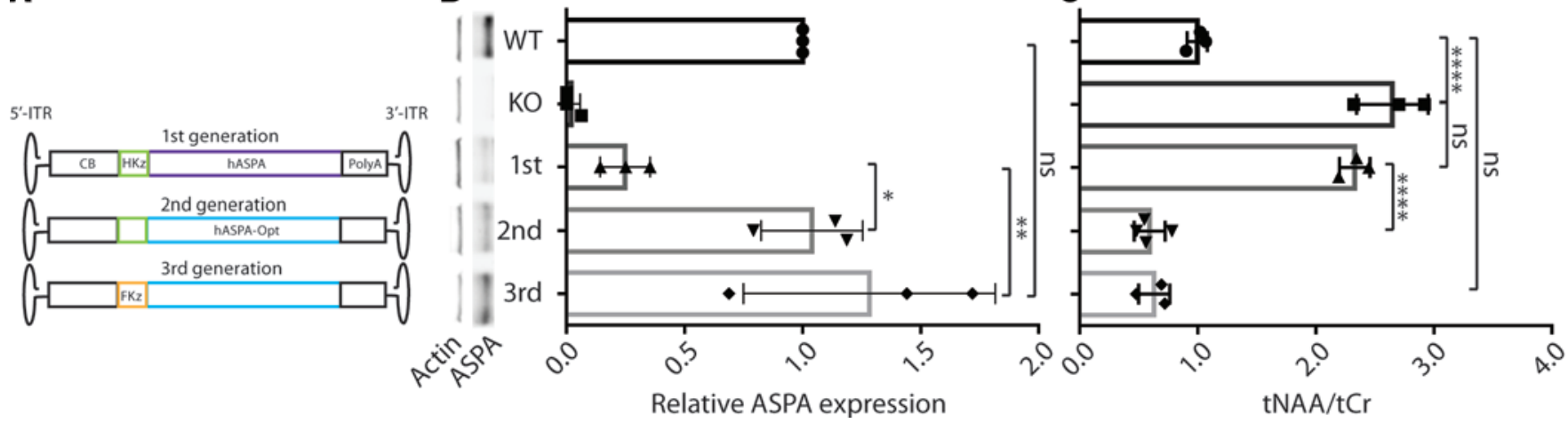

Figure 1. Optimized gene expression cassette rescues normal ASPA protein and NAA levels in Canavan disease knockout (CD KO) mice. (A) Three expression cassettes were cloned carrying either half (HKz) or full Kozak (FKz) sequence and the WT human aspartoacylase (hASPA) cDNA or a codon-optimized (Opt) hASPA. ITR, inverted terminal repeat. (B) Mice were treated at P1 via facial vein with $4 \times 10^{11}$ genome copies of recombinant adeno-associated virus 9 (rAAV9) carrying a 1st, 2nd, or 3rd generation expression cassette. Western blotting of the brains 42 days after treatment shows relative ASPA expression normalized to actin and WT; due to early lethality, untreated CD KO mice were used at P25 as control ( $n=3$ each). (C) Cerebral $N$-acetylaspartate (NAA) levels of treated CD KO mice were quantified using magnetic resonance spectroscopy in living mice at P42; untreated CD KO mice were used at P25 as control $(n=3-4)$. Displayed are total NAA (tNAA) over total creatine ( $\mathrm{tCr}$ ). Statistical analysis was performed using 1-way ANOVA with multiple comparison correction. Data are presented as the mean $\pm \mathrm{SD}, n=3 .{ }^{*} P<0.05,{ }^{* *} P<0.01$, ${ }^{* * *} P<0.0001$. ns, not significant.

impairment within the first month of life. We found that at 1 month of age, 2nd generation-treated CD KO mice performed as well as WT controls on accelerated rotarod (Figure 2A). To our surprise, the 3rd generation treatment group displayed a super-mouse phenotype, significantly outperforming WT mice for the entire 1-year duration of the study (Figure 2A, Supplemental Figure 4, and Supplemental Videos 1 and 2) In order to assess ataxia and the ability to balance, $2 \mathrm{nd}$ and $3 \mathrm{rd}$ generation-treated mice were evaluated on balance beam, showing full recovery as well (Figure 2B and Supplemental Figure 4). This was paralleled by the performance on inverted screen and was persistent throughout the study (Figure $2 \mathrm{C}$ and Supplemental Figure 4). Finally, to determine the long-term ability to rescue spatial/working memory, mice were tested on T maze at 1 year, performing as well as WT control mice (Supplemental Figure 5).

Efficient hASPA gene delivery to the CNS persistently eliminates neuropathology and normalizes NAA levels. To determine if the phenotypic rescue of psychomotor function was supported by brain pathology and NAA biomarker levels, we assessed living mice from all 3 treatment groups at 1 year of age. First, T2 MRI showed strong hyperintensities of 1st generation-treated mice, particularly in the thalamus, midbrain, and cerebellum (Figure $3 \mathrm{~A}$ ). In contrast, 2nd generation-treated mice showed only a mild increase in $\mathrm{T} 2$ signals in the midbrain, appearing otherwise similar to WT mice. Importantly, comparison between WT and 3rd generation-treated mice revealed no difference on T2 MRI, which was also supported by neuropathology analysis (Figure 3B and Supplemental Figure 6). While some regions of the CNS (e.g., thoracic spinal cord) showed similar patterns between WT and all 3 treatment groups, the brain regions with the strongest T2 signal also corresponded with the most severe vacuolization in the brain sections of 1st generation-treated mice (Figure 3, A and B, and Supplemental Figure 7). When 1-year-old mice of all treatment groups were subjected to MRS for NAA quantification, 1st generation-treated mice showed a significantly higher NAA signal than WT mice, while NAA levels of 2nd and 3rd generation-treated mice were normalized (Figure $3 C)$. This strongly suggests that the new-generation gene therapies are significantly more efficacious and are able to normalize NAA biomarker levels, a finding that is further supported by normalized NAA levels in urine, as measured by mass spectrometry (Figure 3D).

Astrocyte-specific ASPA expression is sufficient for generating the super-mouse phenotype. Motor function evaluation raised the critical question of how our 3rd generation gene therapy accomplished performance enhancement beyond WT control animals. We found no reported phenotypic differences between patients who are heterozygous or homozygous for the WT ASPA allele, implying that oligodendroglial ASPA and its associated NAA catabolism might not cause dose-dependent behavioral variations within the physiological range. In addition, most rAAVs including rAAV9 poorly transduce oligodendrocytes (35), which prompted us to hypothesize that $h A S P A$ transgene expression from non-oligodendrocyte glial cells might have contributed to the super-mouse phenomenon seen on accelerated rotarod. If this were the case, WT mice should respond to ASPA supplementation with increased motor performance as well. To test this hypothesis, we treated 

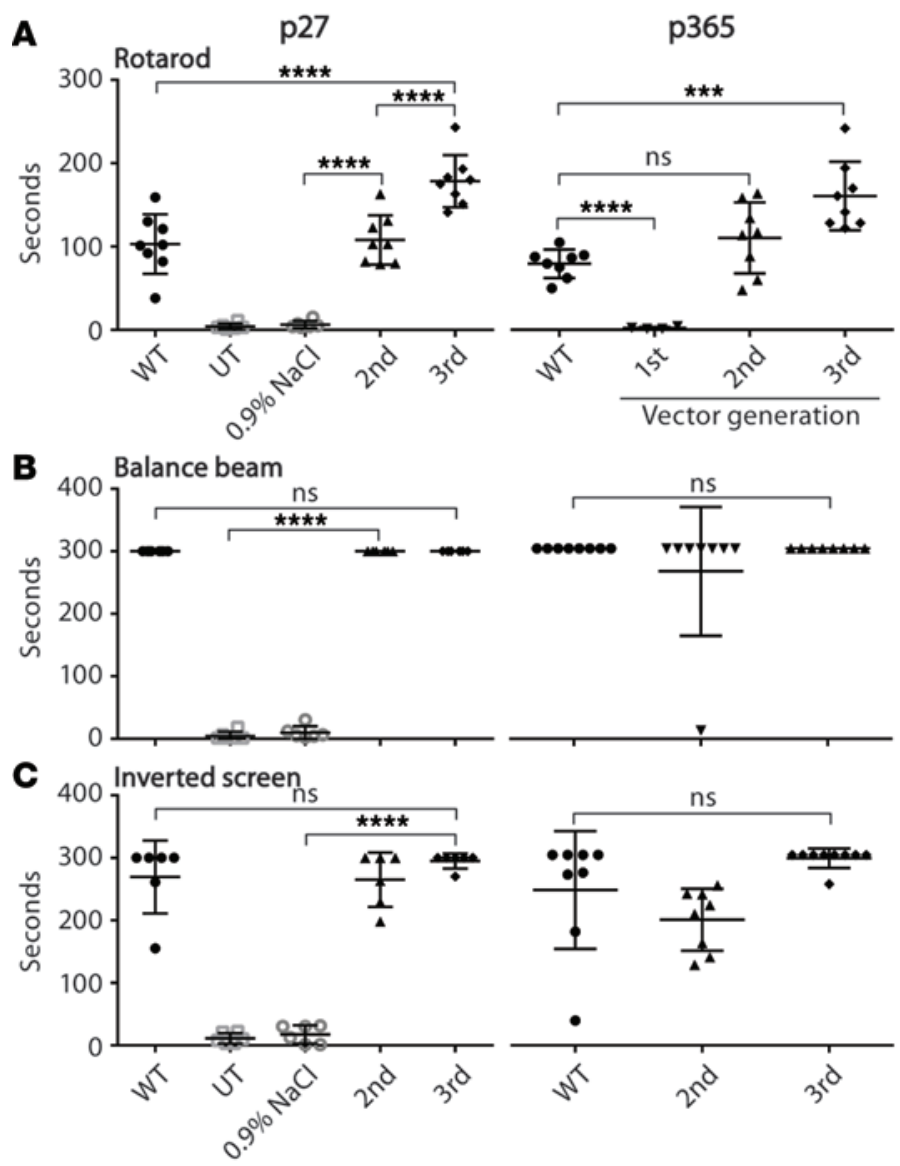

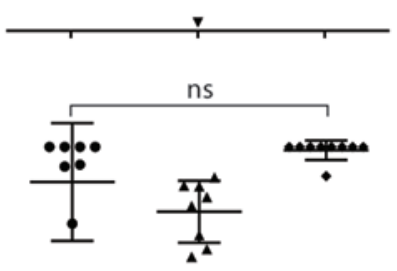

Figure 2. Optimized gene replacement therapy normalizes motor function in Canavan disease knockout (CD KO) mice. Each assay was performed with an independent group of CD KO mice. Mice $(n=24)$ were treated at $\mathrm{P} 1$ with $4 \times 10^{11}$ genome copies into the facial vein using the 2 nd or 3rd generation gene therapy and divided into 3 groups ( $n=8$ each) for each motor function assay. Shown are testing time points at P27 and P365 (see Supplemental Data for additional time points). (A) On accelerated rotarod, mice were trained for 2 consecutive days prior to the testing day. (B) Balance beam tests for deficits in equilibrium sense and for ataxia. Cut-off time point was 300 seconds. (C) Muscle/grip strength was tested on inverted screen for up to 300 seconds. Statistical analysis was performed using 1-way ANOVA with multiple comparison correction. Data are presented as the mean $\pm S D$; for all motor assays, $n=6-8$ at P27 and $n=8$ at P375. ${ }^{* *} P<0.001,{ }^{* * *} P<0.0001$. ns, not significant.

WT animals with 3rd generation gene therapy. Initially, treated WT mice showed no difference on accelerated rotarod at P27, but began significantly outperforming untreated WT controls at P90 to 1 year of age, suggesting that supplementing the non-ASPAexpressing cells with ASPA by gene transfer might contribute to this observed enhanced phenotype (Supplemental Figure 4).

To further define the CNS cell type contributing to the supermouse phenotype, we paired our optimized hASPA construct with a partial human glial fibrillary acidic protein (phGFAP) promoter. First, we confirmed the astrocyte specificity of the phGFAP promoter by expressing enhanced green fluorescence protein (EGFP), which showed colocalization with GFAP-positive cells (astrocytes), but not with myelin basic protein-positive (MBP-positive; oligodendrocytes) or neuronal nuclei-positive (NeuN-positive; neurons) cells (Figure 4, A and B, and Supplemental Figure 7). We next treated neonatal CD KO mice with codon-optimized rAAVphGFAP-hASPA (rAAVphGFAP- $h A S P A-O p t)$. Treated mice displayed the same growth curve as the group treated with ubiquitously expressed 3rd generation gene therapy (Figure 4C). Interestingly, the performance of these mice on accelerated rotarod was significantly increased over WT mice at all testing time points (P27 and P90; study ongoing), similar to what was seen with the 3rd generation vector for ubiquitous hASPA expression (Figure 4D). This suggests that astrocyte-restricted hASPA expression contributes substantially to the enhanced performance on accelerated rotarod. As seen before, other less stringent motor function tests did not show a difference compared with WT mice (Figure 4D). Importantly, rAAVphGFAPhASPA-Opt-treated mice performed better than untreated control mice on T maze, but showed no difference in spatial/working memory function versus WT animals (Figure 4E). This suggests that mice with increased motor performance have normal spatial/working memory function on T-maze testing. In addition, MRI and MRS showed normalization of T2 signals and NAA levels in the brains of mice receiving astrocytespecific gene therapy (Figure 4, F and G), suggesting that astrocyte-restricted hASPA expression alone is capable of creating an alternative metabolic sink for NAA and is rescuing neuropathology and biomarker expression in the $\mathrm{CD}$ mice. In addition, Western blotting showed supraphysiologic levels of hASPA after treatment (Figure $4 \mathrm{H}$ ), further supporting the reduction of brain NAA levels. To corroborate that astrocyterestricted hASPA expression can indeed rescue neuropathology, i.e., improve myelination, brain sections were stained with Luxol fast blue for myelin sheaths. The staining pattern was indistinguishable between WT, astrocyte-restricted, and ubiquitously expressed 3rd generation gene therapy-treated animals, and in all cases myelination was recovered compared with untreated CD KO mice (Figure 4I).

Optimized gene therapy achieves efficacious rescue of the $C D$ phenotype at lower doses. One important aspect in translating gene therapy into the clinic is the vector dose, which is relevant to manufacturing burden, costs, and safety. Based on the performance of our treated mice with full-dose $\left(4 \times 10^{11}\right.$ genome copies [GCs] $/$ animal), 3rd generation gene therapy, we opted for 3-fold $\left(1.33 \times 10^{11} \mathrm{GC}\right)$ and 10 -fold $\left(4 \times 10^{10} \mathrm{GC}\right)$ lower doses to compare their therapeutic outcomes. Within the first 4 weeks of life, 3- and 10-fold lower 3rd generationtreated mice showed significantly better weight gain than full-dose and 3-fold lower 1st generation-treated 
A

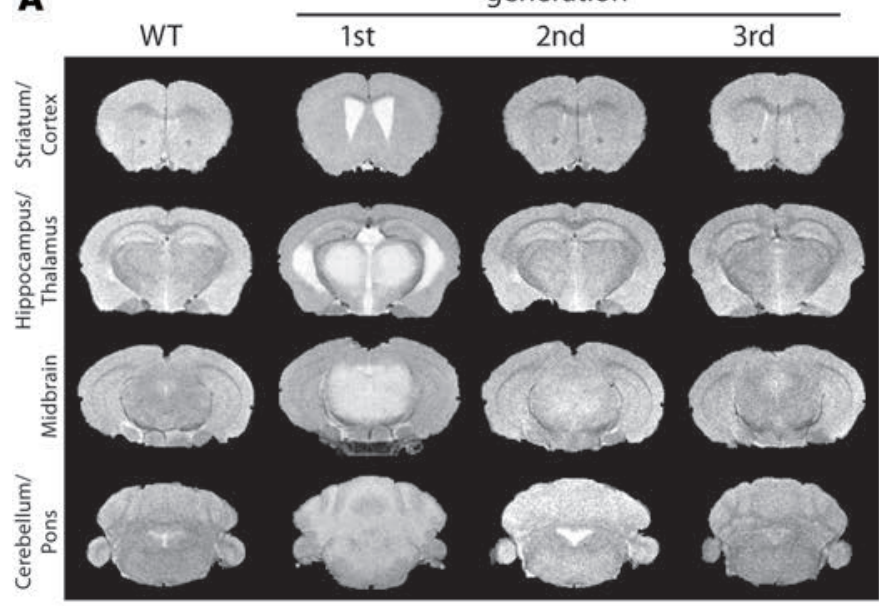

c

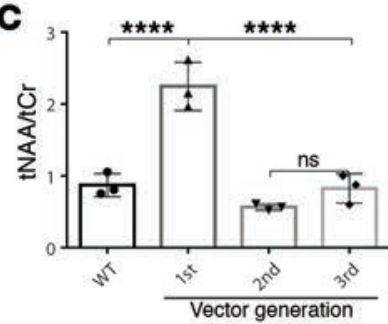

generation

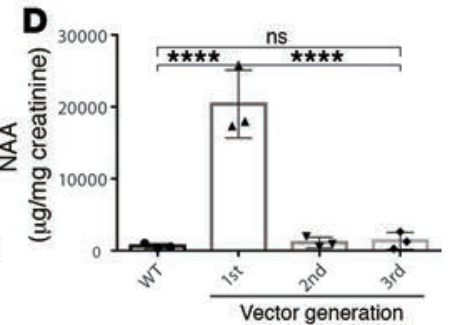

B
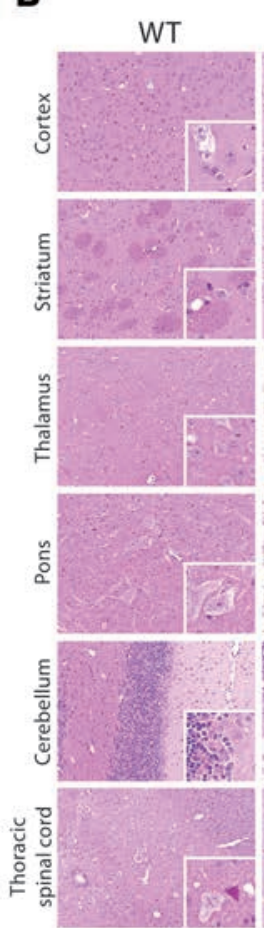

generation

1st 2 nd 3 rd

Figure 3. Optimized gene replacement therapy leads to sustained rescue of neuropathology and biomarker expression. Mice were treated at $\mathrm{P} 1$ intravenously with $4 \times 10^{11}$ genome copies of 1st, 2 nd, or 3rd generation vector. Data from 1 year after treatment are shown in comparison to WT. (A) MRI imaging comparing WT and all 3 vector generations. (B) H\&E staining shows degree of neuropathologic changes (original magnification, $\times 10$; insets, $\times 40)$. (C) Magnetic resonance spectrometry (MRS) analysis of brain total $N$-acetylaspartate (tNAA) levels normalized against total creatine (tCr) at 1 year of age. (D) NAA levels of urine normalized against creatinine at 1 year of age as quantified by MRS. Statistical analysis was performed using 1-way ANOVA with multiple comparison correction. Data are presented as the mean $\pm \mathrm{SD}, n=3$. ${ }^{* * *} P<0.0001$. ns, not significant.

mice (Supplemental Figure 1 and Supplemental Figure 8 insets). This was further shown over the course of the entire study period for the 3-fold lower dose 3rd generation group, which paralleled the weights of WT animals. In contrast, 3-fold lower dose 1st generation-treated mice declined starting at 16 weeks of age (Supplemental Figure 8). Again, the study mice were subjected to MRI and MRS for in vivo CNS assessment, but at an earlier time point (P25). T2 sequences showed hyperintensities particularly in thalamus, midbrain, cerebellum, and brain stem of untreated mice (Figure 5A). This signal was reduced in a dose-dependent manner, with the 3-fold lower dosing group showing the least hyperintense signal. As a control, WT and full-dosetreated CD mice showed normalized T2 signals and were indistinguishable from each other (Figure 5A). The MRI findings from the dose down-escalation study were also reflected in the NAA quantification by MRS (Figure 5B). This was further supported by neuropathology analysis, showing a dose-dependent distribution of vacuoles with a similar pattern in 1st generation full-dose-treated mice and 10-fold lower 3rd generationtreated mice (Figure 5C and Supplemental Figure 9). Finally, mice were tested on accelerating rotarod, balance beam, and inverted screen. At P27, 10-fold lower 3rd generation-treated mice performed as well as WT mice on rotarod and balance beam, but not as well on inverted screen test (Figure 5D). Over time, this lowdose treatment group showed deterioration of motor function by P90 (data not shown). In contrast, at P27, the 3-fold lower dose could restore the motor functions of CD KO mice to the levels of WT mice, as measured by all 3 aforementioned tests. By P90, these mice were still performing as well as WT mice on accelerating rotarod and balance beam, but not on inverted screen (Figure 5D). These data indicate that the 3rd generation gene therapy is significantly more potent, even at lower doses, than our 1st generation gene therapy.

Vector genome requirements to mitigate neuropathology vary among CNS regions. To obtain further insight into CNS-region-specific pathology and rAAV transduction, inferring that expression cassette optimization correlates with higher hASPA protein expression (Figure 1B), 11 CNS regions were analyzed. No significant differences in vector GC number per diploid cell between full-dose 1st and 3rd generation treatment groups 

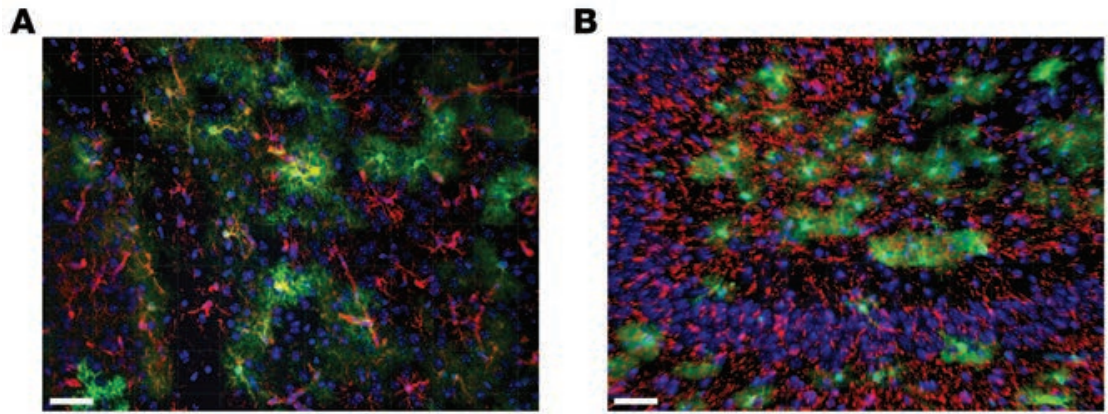

D
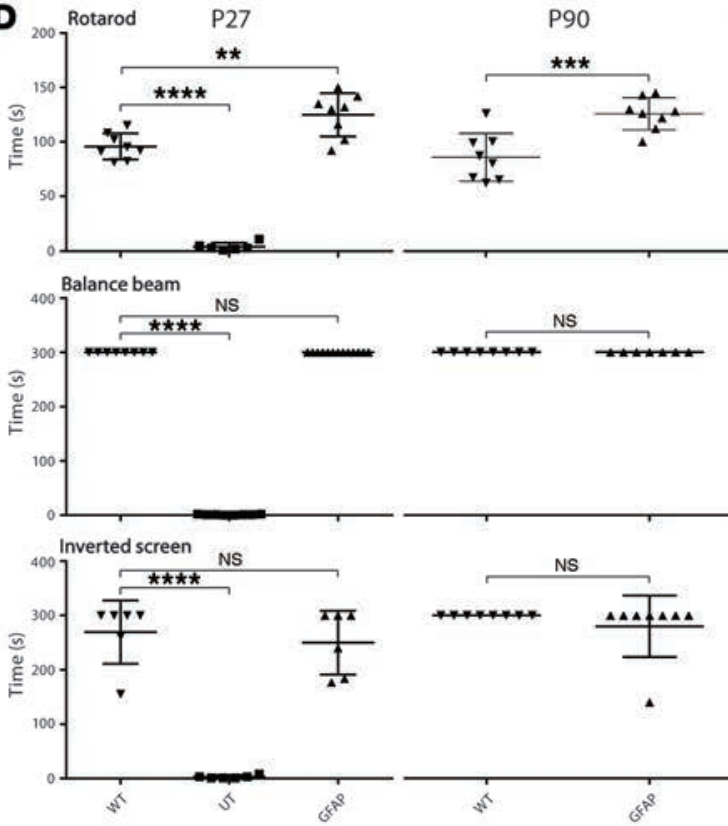

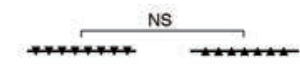

G
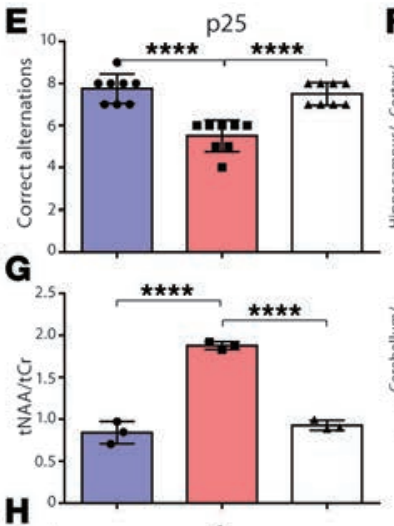

H
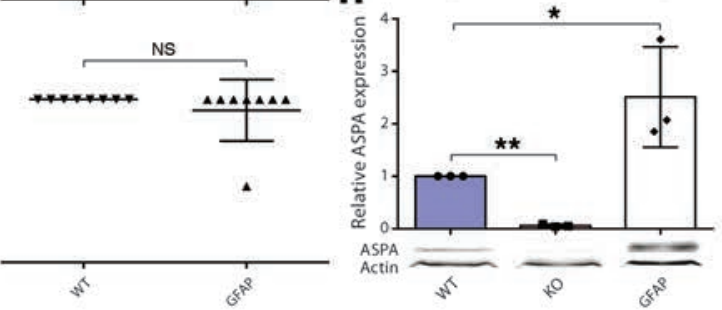

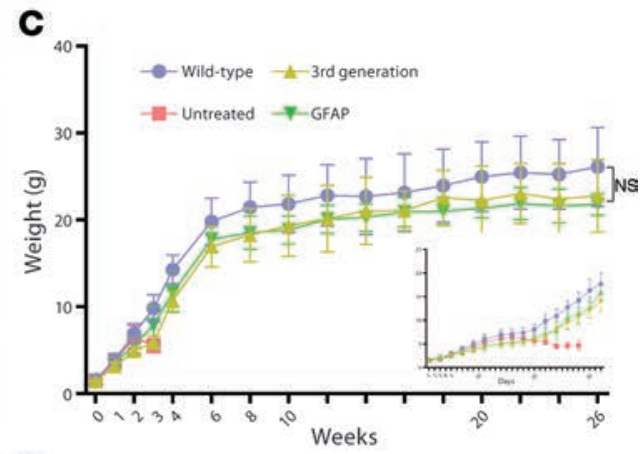

$\mathbf{F}$
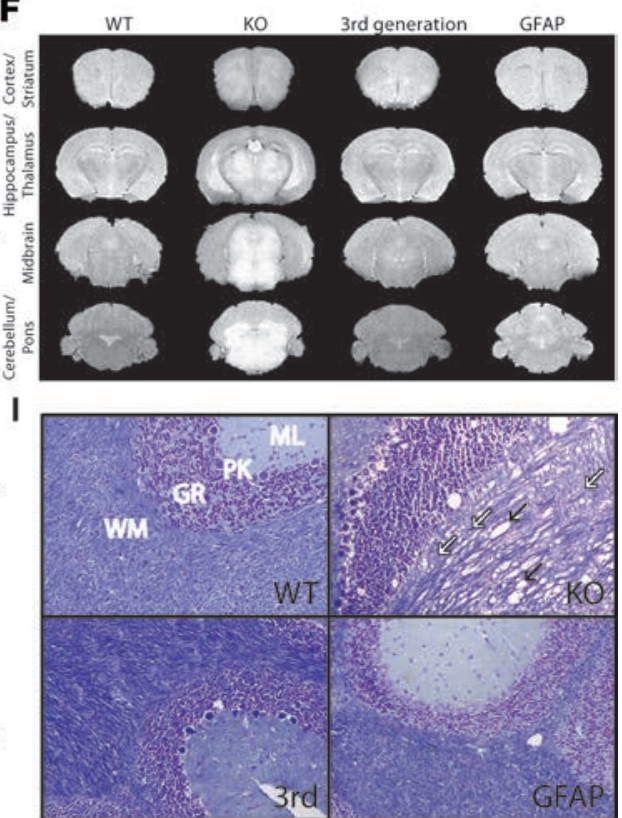

Figure 4. Astrocyte-restricted hASPA expression rescues motor function, neuropathology, and biomarker expression in Canavan disease knockout (CD KO) mice. Mice were treated intravenously at P1 with $4 \times 10^{11}$ genome copies of 3rd generation gene therapy containing either a ubiquitous or a partial human glial fibrillary acid protein (phGFAP) promoter. (A and B) Brain sections with astrocyte-restricted EGFP expression that colocalizes with GFAP-positive (red in A) cells but not with myelin basic protein-positive (MBP, red in B) cells $(n=3)$; high magnification of the same images provided in Supplemental Figure 7, C and D. Scale bars: $50 \mu \mathrm{m}$. (C) Weights of WT mice vs. mice treated with ubiquitous or astrocyte-restricted human aspartoacylase (hASPA) expression ( $n=8-10$ ) by day (inset) and by week. (D) Motor function (accelerated rotarod, balance beam, and inverted screen) and cognitive (T maze; E) tests of mice with astrocyte-restricted hASPA expression ( $n=6-8)$. UT, untreated. (F) MRI shows T2 signal pattern of ubiquitous vs. astrocyte-restricted hASPA expression in comparison to WT and untreated (KO) mice (P25; $n=3)$. (C) Magnetic resonance spectrometry of brain shows brain $N$-acetylaspartate (NAA) levels and (H) Western blotting shows brain ASPA expression (P25; $n=3$ ). (I) Luxol fast blue staining of myelin shows a reduction of myelin fibers (white arrows) and the presence of vacuoles (black arrows) in the cerebellar white matter of CD KO mice vs. mice treated with ubiquitous or astrocyte-restricted hASPA expression ( $225 ; n=3$; original magnification, $\times 10)$. GFAP, GFAP promoter-driven hASPA; ML, molecular layer; PK, Purkinje cell layer; GR, granular layer; WM, white matter. Statistical analysis was performed using 2-way ANOVA with multiple comparison correction for $\mathbf{C}$ and 1-way ANOVA with multiple comparison correction was used for $\mathbf{D}, \mathbf{E}, \mathbf{G}$, and $\mathbf{H}$. Data are presented as the mean \pm SD. ${ }^{*} P<0.05,{ }^{* *} P<0.01,{ }^{* *} P<0.001,{ }^{* * *} P<0.0001$. ns, not significant.

were detected, supporting our hypothesis that increased hASPA protein expression from the optimized expression cassette, but not improved vector genome delivery, was responsible for the significant therapeutic improvement of the 3rd generation-treated mice (Figure 1B and Figure 6). When regional distribution of vector genome was ranked, cortex showed the highest and cerebellum the lowest rAAV GC number per cell (Figure 6). In relation to neuropathology, this suggests that certain brain regions have different therapeutic thresholds to achieve complete mitigation (Figure 3 and Supplemental Figure 9). Particularly the cerebellum showed minimal response comparing full-dose 1st generation to 10- and 3-fold lower dose 3rd generation gene therapy (Supplemental Figure 9). However, upon full-dose 3rd generation treatment, complete rescue was achieved with rAAV GC numbers lower than in any other CNS region (Figure 6 and Supplemental Figure 9). 


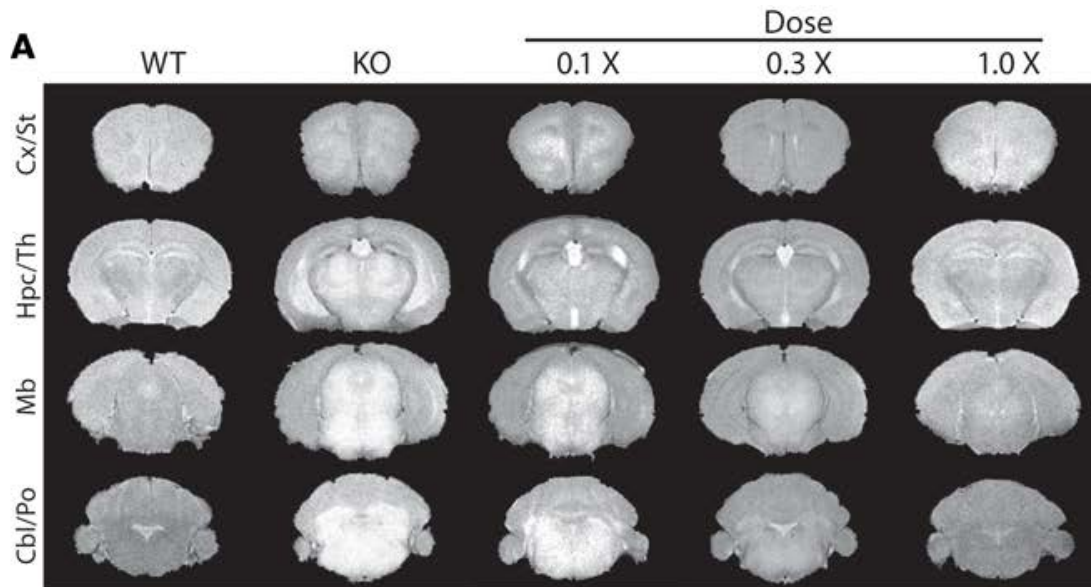

\section{B}

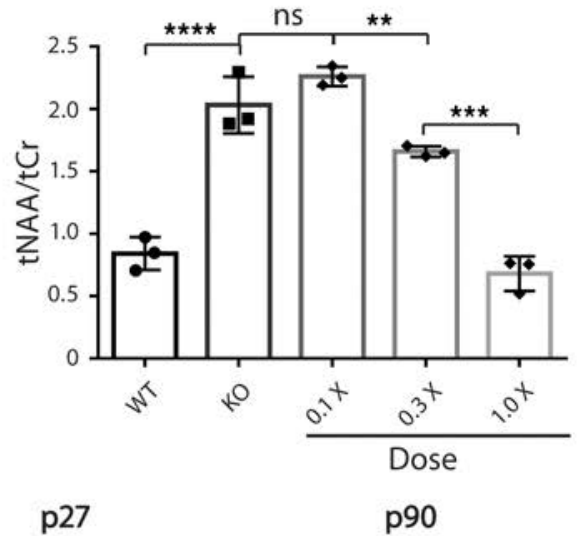

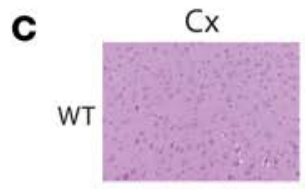
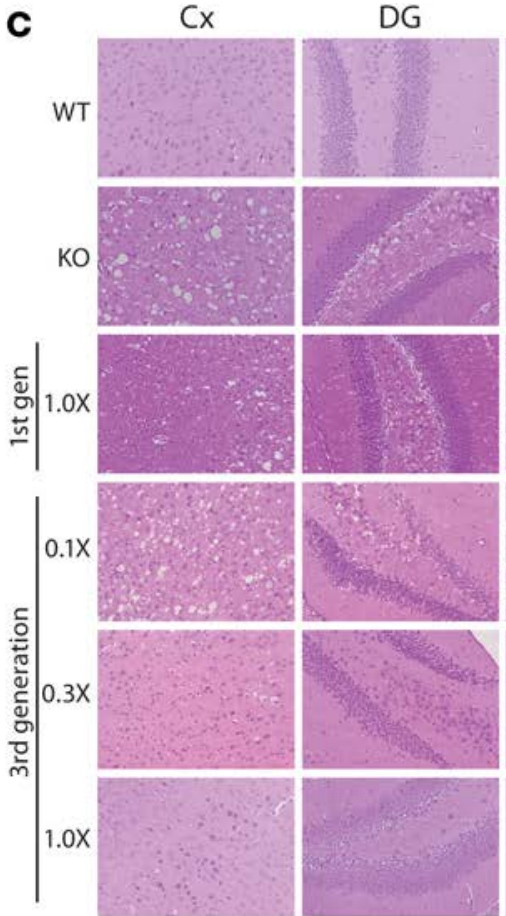

Th
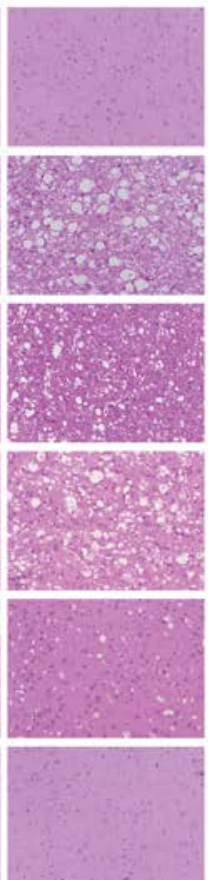

Po
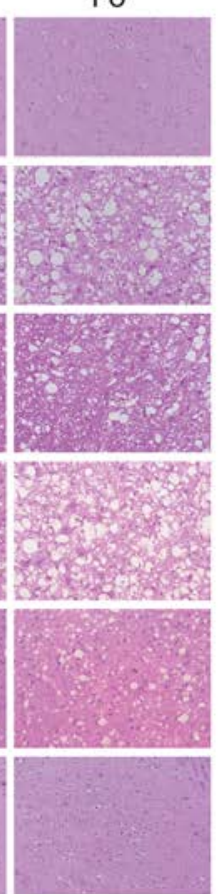

D
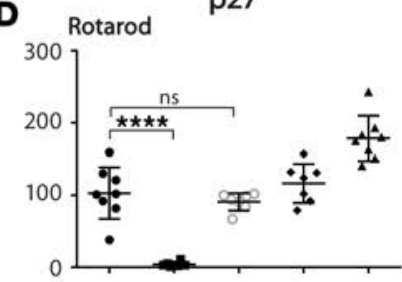

Balance beam
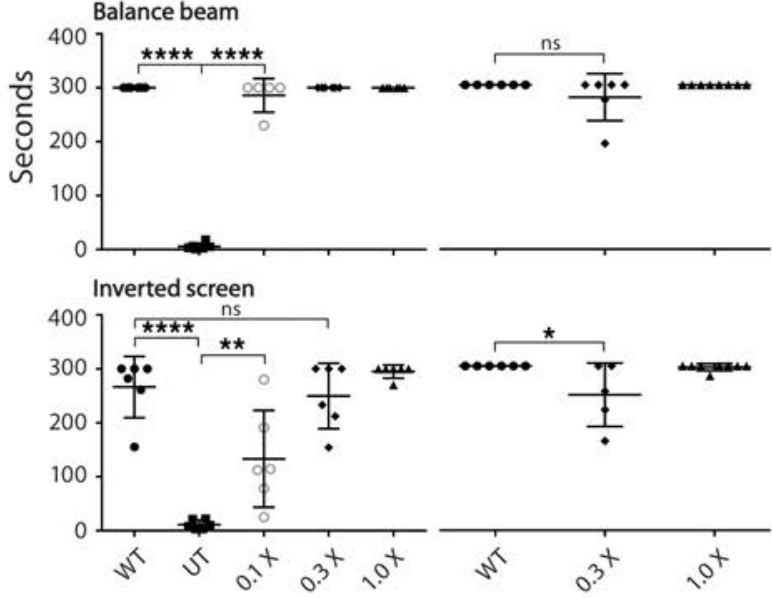

Figure 5. Dose reduction of 3rd generation therapy achieves efficacious disease rescue in Canavan disease knockout (CD KO) mice. CD KO mice were treated at $\mathrm{P} 1$ via facial vein with either $4 \times 10^{10}(0.1 \mathrm{X}), 1.33 \times 10^{11}(0.3 \mathrm{X})$, or $4 \times 10^{11}(1 \mathrm{X})$ genome copies of 3rd generation gene replacement therapy. $(\mathrm{A}) \mathrm{MRI}$ T2 sequences of different brain regions are shown with WT and untreated (KO) control mice. All mice were imaged at P25 ( $n=3)$. (B) Total N-acetylaspartate (tNAA) levels of the same mice as in A were quantified by magnetic resonance spectrometry at P25 and normalized against total creatine (tCr) ( $n$ = 3). (C) Dose-dependent neuropathology assessed by H\&E staining of brain regions of mice at P25 treated with 3rd generation or full-dose 1st generation vectors. WT and KO were used as controls $(n=3$; original magnification, $\times 10)$. (D) Dose-dependent motor function was assessed on rotarod, balance beam, and inverted screen at P27 and P90 ( $n=6-8)$. Cx/St, cortex/striatum; Hpc/Th, hippocampus/thalamus; Mb, midbrain; Cbl/Po, cerebellum/pons; Cx, cortex; DG, dentate gyrus; Th, thalamus; Po, pons. Statistical analysis was performed using 1-way ANOVA with multiple comparison correction. Data are presented as the mean $\pm \mathrm{SD}$. ${ }^{*} P<0.05,{ }^{* *} P<0.01,{ }^{* *} P<0.001$, ${ }^{* * *} P<0.0001$. ns, not significant.

High-field neuroimaging enables noninvasive monitoring and prediction of therapeutic outcomes. One critical aspect of CD is the loss of myelin structures in the CNS. The myelin stain Luxol fast blue showed widespread vacuoles and reduced myelin in untreated CD KO mice (Supplemental Figure 10). In contrast, myelin structures in WT and full-dose 3rd generation-treated mice were indistinguishable (Supplemental Figure 10). To evaluate if these findings can be monitored in the living mouse, high-field DTI was applied to assess myelin fiber tracts in the corpus callosum (CC) and the external capsule (EC). First, proof-of-concept tractography to obtain an overall assessment of fiber tract morphology showed substantially altered and shortened interhemispheric tracts of the CC in untreated mice, whereas WT and 3rd generation-treated mice were indistinguishable (Figure 7A). For quantitative assessment, fractional 


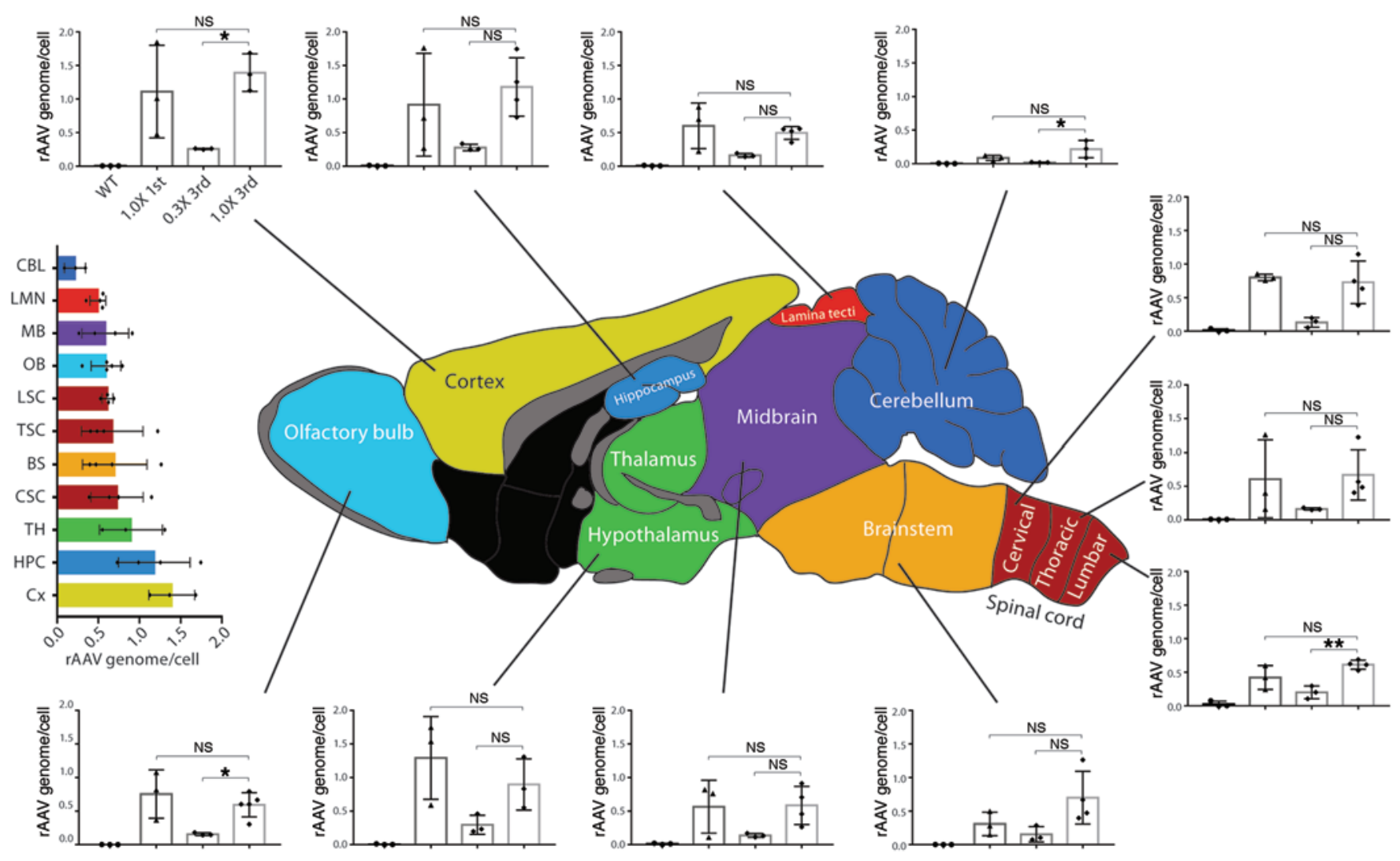

Figure 6. Recombinant adeno-associated virus (rAAV) vector genome copy numbers vary among brain regions of treated Canavan disease knockout (CD KO) mice. Eleven different regions of the CNS were analyzed for rAAV9 vector genome copy (GC) number per cell. Displayed is a schematic drawing of a sagittal section of mouse brain with 11 brain regions, including spinal cord, labeled and highlighted. Vector GC number is shown per diploid cell (rAAV genome/cell) for WT control, and 1-year-old CD KO mice treated with full-dose 1st generation, and full and 3-fold lower dose 3rd generation gene therapy. On the left side, a ranking of the rAAV genome/cell of the 3rd generation treated is shown. $1.0 \mathrm{X} 1 \mathrm{st}=4 \times 10^{11} 1 \mathrm{st}$ generation; $0.3 \times 3 r d=1.33 \times 10^{11} \mathrm{GC}$ 3rd generation; $1.0 \mathrm{X}$ 3rd $=4 \times 10^{11} \mathrm{GC}$ 3rd generation. CBL, cerebellum; LMN, lamina tecti; MB, midbrain; OB, olfactory bulb; LSC, lumbar spinal cord; TSC, thoracic spinal cord; BS, brain stem; CSC, cervical spinal cord; TH, thalamus and hypothalamus; HPC, hippocampus; Cx, cortex. Statistical analysis was performed using 1-way ANOVA with multiple comparison correction. Data are presented as the mean \pm SD; $n=3-4 .{ }^{*} P<0.05,{ }^{* *} P<0.01$. ns, not significant.

anisotropy (FA) values of the CC and right and left EC were compared between groups. While there was no significant difference between WT and treated mice, untreated mice differed significantly, supporting the overall impression of the tractography and the value of DTI for noninvasive CNS gene therapy assessment (Figure 7B).

The observation that mice treated with the 3rd generation gene therapy significantly outperformed WT mice, which could not be anticipated based on MRI/MRS or DTI data, prompted us to propose that ubiquitous ASPA expression changes functional connectivity, which might correlate with the motor function phenotype. To test this hypothesis, we performed resting state fMRI (rs-fMRI) on groups of WT, untreated, and treated male mice and analyzed 19 different brain regions (Supplemental Figure 11). Based on T-score analysis, untreated mice showed the most active brain regions, with decreasing activity in WT and treated mice, suggesting that the ASPA-deficient brain might have to engage more brain regions at baseline (Figure 7, C and D). Although the difference between WT and treated mice with regard to overall functional activity was marginal, ubiquitous ASPA expression in the brain might facilitate the communication between brain regions and thus require the engagement of fewer brain regions at baseline (Figure 7, C and D). Correlation analysis between the average number of active brain regions and accelerated rotarod results revealed a negative correlation between overall functional connectivity and accelerated rotarod motor function, suggesting that rs-fMRI might support the predictive correlation of treatment and motor performance $\left(\mathrm{R}^{2}=0.89\right.$; Figure 7, D and F). As control, NAA levels were quantified by MRS (Figure 7E). 
A
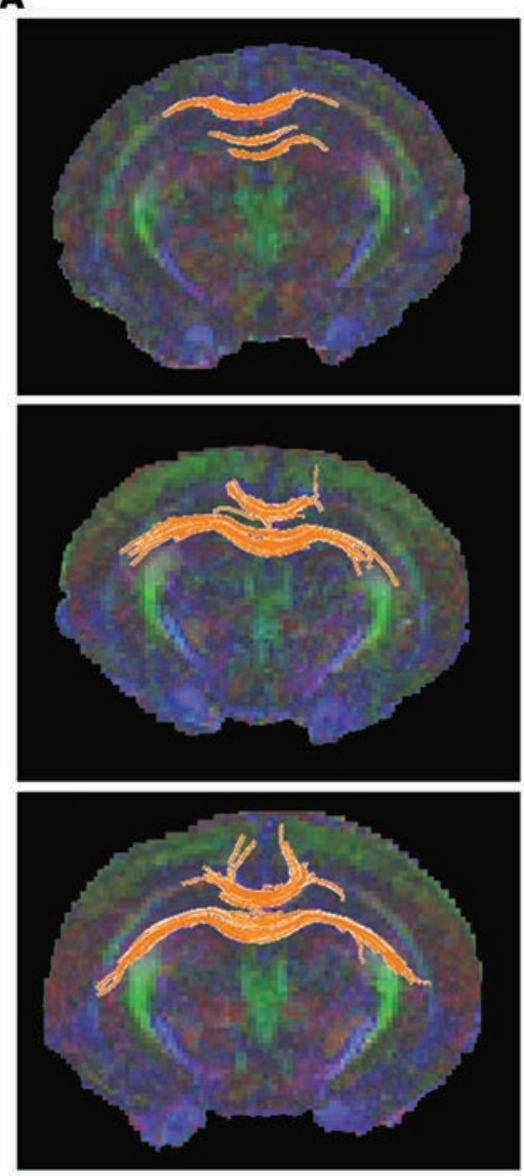

B

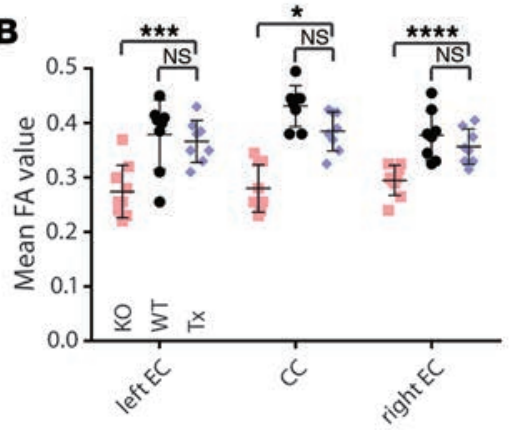

C
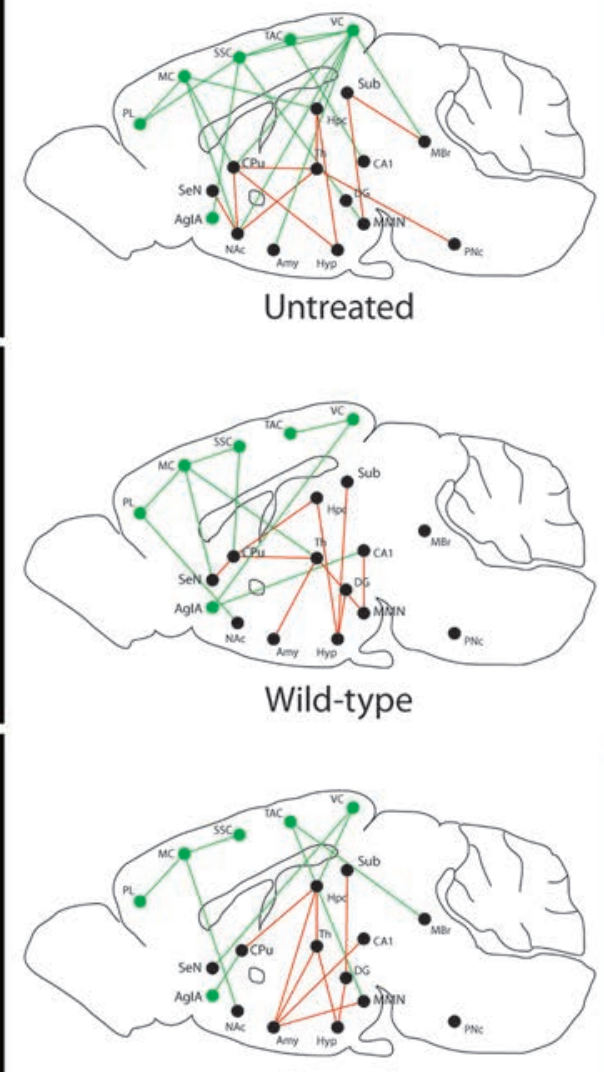

Treated

E

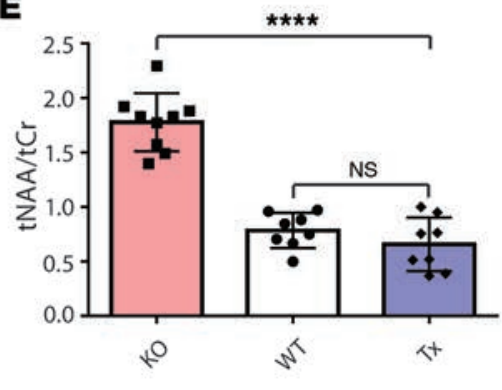

F
D
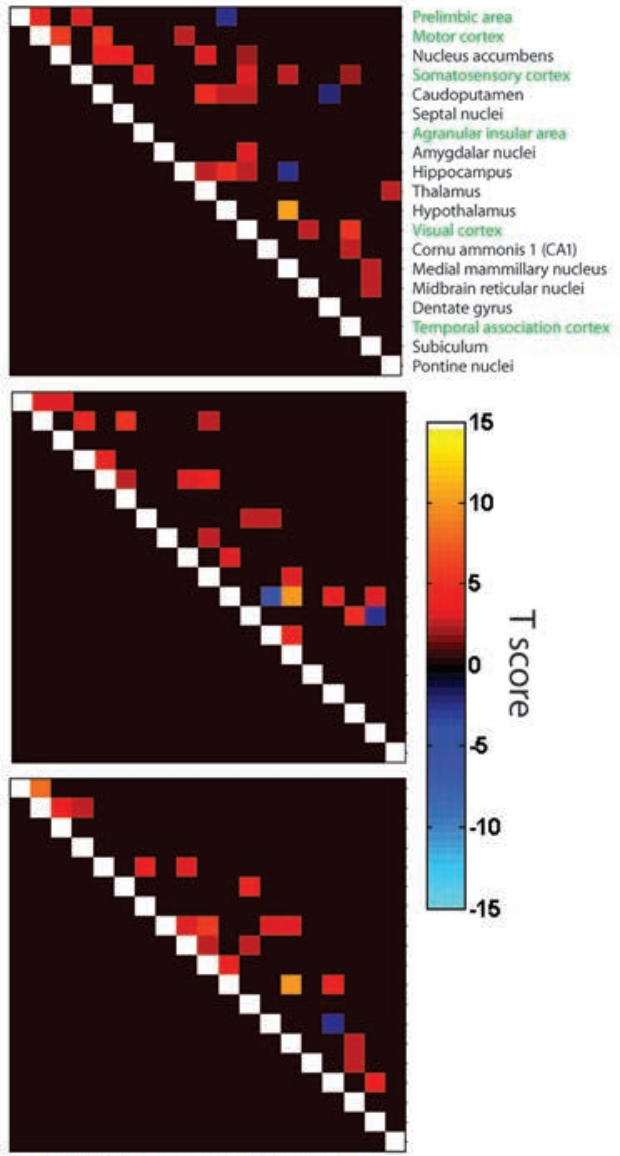

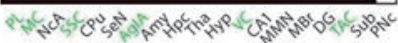

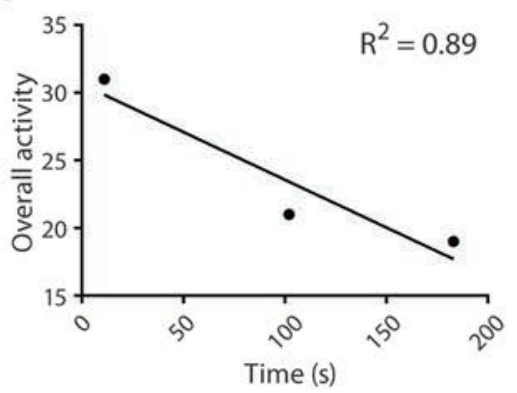

Figure 7. High-field imaging noninvasively evaluates therapy outcomes. Male mice were treated at P1 and imaged at P25, comparing untreated Canavan disease knockout (CD KO) mice, WT mice, and treated (TX) CD KO mice ( $n=8-10$ each). (A) Representative images are shown for tractography of the corpus callosum. (B) Shown are the fractional anisotropy (FA) values for left and right external capsule (EC) and corpus callosum (CC). KO, untreated CD KO mice. (C) Nineteen brain regions were selected to analyze resting state-functional MRI (rs-fMRI) results and mapped on a sagittal brain map. Green and black dots indicate cortical and subcortical brain regions, respectively. Green lines display all connections that involve cortical regions and red lines show subcortical connections only. (D) T-score statistics of rs-fMRI are shown that indicate differences between corresponding brain regions and overall brain activity (displayed in C). (E) Total $\mathrm{N}$-acetylaspartate (tNAA) normalized against total creatine (tCr) was measured in all mice that underwent diffusion tensor imaging and rs-fMRI imaging. (F) Overall functional connectivity (untreated 31, WT 21, treated 19) was correlated to the average accelerated rotarod performance in seconds (Figure 3A, P27) and analyzed via linear regression analysis $\left(R^{2}=0.89\right)$. PL, prelimbic area; MC, motor cortex; NAc, nucleus accumbens; SSC, somatosensory cortex; CPu, caudoputamen; SeN, septal nuclei; AgIA, agranular insular area; Amy, amygdalar nuclei; Hpc, hippocampus; Th, thalamus; Hyp, hypothalamus; VC, visual cortex; CA1, cornu ammonis; MMN, medial mammillary nucleus; DG, dentate gyrus; TAC, temporal association cortex; Sub, subiculum; PNc, pontine nuclei. Statistical analysis was performed using 1-way ANOVA with multiple comparison correction for $\mathbf{B}$ and $\mathbf{E}$ and correlation analysis for $\mathbf{F}$. Data are presented as the mean $\pm \mathrm{SD}, n=8-10$. ${ }^{*} P<0.05,{ }^{* * *} P$ $<0.001,{ }^{* * *} P<0.0001$. ns, not significant. 


\section{Discussion}

rAAV-based gene replacement therapy for $\mathrm{CD}$ is a promising therapeutic avenue. The early clinical trial demonstrated safety for intracranially administered $\mathrm{rAAVh} A S P A$, and a proof-of-concept rAAV-based gene therapy (1st generation) study in mouse showed that i.v. delivered rAAV can preclinically impact this leukodystrophy $(22,33)$. However, both studies were unable to demonstrate the desired therapeutic efficacy; i.e., early, complete, and sustained disease rescue. Several reasons for the low efficacy were discussed, including the strength of the expression cassette, route of delivery, and rAAV serotypes. Fortunately, the discovery of a plethora of new AAVs that cross the blood-brain barrier and achieve widespread transduction of the CNS allows for the development of new therapeutic strategies, as well as more detailed studies of NAA function (36-38). Among these vectors, we previously evaluated rAAV9, rh.8, and rh.10 using our 1st generation expression cassette for the treatment of the CD KO mouse, with each vector showing similar efficacy (33). In the current study, we demonstrate the efficacy of our optimized hASPA gene replacement therapy that not only provides an excellent tool to study the function of NAA but also holds great promise to treat $C D$ patients.

Our results demonstrate that the 3rd generation gene therapy achieves early and sustained disease rescue with approximately 10 -fold higher potency as shown by dose escalation. Although the 10-fold lower dose could not achieve sustained rescue, it might be beneficial for milder phenotypes of the disease and useful for determining the dependence of cellular function on NAA turnover rate. Detailed analysis of $11 \mathrm{CNS}$ regions showed that expression cassette optimization and not rAAV genome delivery was responsible for the improved therapeutic efficacy. Assessment of neuropathology revealed that the phylogenetically older regions of the CNS, such as thalamus, pons, and cerebellum, appeared to be most affected and also showed the most obvious response to treatment. Interestingly, this brain-regiondependent analysis revealed that not all regions required the same amount of rAAV vector genome in order to achieve normalization of neuropathology. For example, the pathology in the cerebellum showed severe vacuolization, but also the lowest vector GC number per diploid cell of all brain regions analyzed. Nevertheless, the pathology in cerebellum was completely rescued at 1 year of age upon $3 \mathrm{rd}$ generation treatment, indicating that even small amounts of ASPA are therapeutically sufficient. In contrast, the thalamus displayed severe vacuolization, but required higher levels of vector genome to achieve complete normalization.

It is commonly accepted that NAA synthesis occurs in neurons and is hydrolyzed in oligodendrocytes (39), with no detected activity in astrocytes (5). Nevertheless, in accordance with the molecular water pump theory that NAA functions as an osmolyte, it was hypothesized that astrocytes play a role in $\mathrm{CD}$ via increased $N$-acetylaspartylglutamate (NAAG) and its interaction with astrocytic glutamate receptors and subsequent extracellular breakdown of NAAG and liberation of NAA, leading to osmotic shifts and intracerebral water accumulation (40). Following the paradigm of oligodendroglial ASPA, gene replacement therapy for $\mathrm{CD}$ has focused on ASPA restoration in oligodendrocytes. Here we show that astrocyte-restricted expression is sufficient to achieve disease rescue in the CD KO mouse, and substantially contributes to the super-mouse phenomenon. Although a mechanistic explanation for this contribution of astrocytes to the super-mouse phenotype is currently missing, the function of astrocytes in neurotransmitter or metabolic homeostasis might be one of the underlying causes.

While a performance-enhancing effect in humans is ethically prohibitive, the super-mouse effect can be normalized in mice by lowering the dose of rAAV with the potential clinical implication that a lower dose in humans might be sufficient to achieve disease rescue. In addition, astrocyte-restricted transgene ASPA expression argues against the common assumption that orthotropic gene function is mandatory for clinically meaningful therapeutic efficacy. We postulate a metabolic sink theory for CD in which reduction of NAA levels independent of the metabolizing glial cell type is therapeutic, since neuronal expression of ASPA has been shown to have limited therapeutic effect (32). This theory implies that either a compensating mechanism for oligodendroglial NAA exists as inferred from the work of others $(41,42)$ or the function of NAA can be rerouted to astrocytes to provide essential metabolites to oligodendrocytes (43). Given the metabolic connections among glial cells, it would not be surprising if metabolic support is not only provided from glia to neurons but also among glial cells, potentially establishing the NAA metabolism in astrocytes to provide aspartate and acetate to oligodendrocytes (44). In that context, the efficacy of our 3rd generation $h A S P A$ expression system highlights its general usefulness for the study of NAA metabolism in different cells and tissues. 
In order to assure that GFAP-promoter-driven expression of hASPA is restricted to astrocytes, we used a GFAP-EGFP approach. Nevertheless, there is a possibility that the GFAP promoter drives hASPA expression in the progenitor cells that give rise to neurons, astrocytes, and oligodendrocytes (45), which could lead to ASPA expression in oligodendrocytes. However, it is unlikely that this potential contribution would be sufficient to rescue the CD KO mouse owing to the episomal nature of the rAAV genome, its subsequent dilution upon cell division, and the relative specificity of the GFAP promoter for astrocytes rather than oligodendrocytes, which would prevent sustained expression in the latter cell type. We also did not explore the possibility that astrocyte-targeted expression of hASPA improves motor learning, thus resulting in increased motor performance.

In the current study, we showed that high-field neuroimaging is an effective means to noninvasively assess the response to hASPA delivery. This approach demonstrated that the myelin defects seen on neuropathology are reflected in DTI of the CC, where affected mice show vacuoles and decreased FA values and fiber length. To advance this approach, longitudinal studies that track the response to treatment over time in both mouse models and patients are needed. Furthermore, the correlation between anesthesia, imaging time, and resolution necessitates determining the best risk-benefit and cost-result ratio in animals to translate this methodology to patients. Nevertheless, our proof-of-concept DTI study demonstrates that neuroanatomical changes due to disease or treatment can be detected and quantified noninvasively. Rs-fMRI complemented DTI by measuring the activation of brain regions, thus allowing for conclusions on pathophysiology and treatment. Others have shown that chemokine receptor deficiency leads to decreased functional connectivity in mice and correlates with altered social behavior (20). In our study, increased functional connectivity was observed in the CD KO mice and correlated with decreased motor performance, while treated mice showed decreased overall functional connectivity, but increased rotarod performance beyond that of WT mice. In this context, fMRI might detect CNS changes that remain undetected on neuropathology and MRI, as seen with the increased motor performance and decreased overall functional connectivity of 3rd generation-treated mice. Nevertheless, one challenge in employing fMRI is to determine how this difference in functional connectivity and motor performance is realized on an anatomical level. One could hypothesize that the reduced functional connectivity of systems executing motor function, e.g., caudoputamen, which is part of the basal ganglia, in the treated mice provides additional capacity to execute motor function and can be recruited under challenging circumstances, e.g., accelerated rotarod. In Parkinson's disease (PD), for example, increased functional connectivity of certain pathways seems to underlie the pathomechanism of PD (17). Furthermore, it might facilitate investigating the role of NAA in cognitive function, as indicated by previous studies $(46,47)$.

Although our understanding of ASPA in peripheral organs is limited $(26,48,49)$ and astrocyterestricted expression shows preclinical disease rescue, clinical translation might benefit from ubiquitous ASPA expression. This is primarily based on the observation of high ASPA expression levels in peripheral organs (3). However, why CNS-restricted ASPA expression cures symptoms in the CD KO mouse or if there is a mechanism of compensation when peripheral organs lack ASPA is still unknown. Despite these remaining questions, our 3rd generation gene therapy holds great promise to study unanswered questions about NAA metabolism, potentially benefitting other CNS diseases, and for clinical translation in the treatment of $\mathrm{CD}$.

\section{Methods}

\section{Animal procedures}

The Aspa KO mouse model was a gift from Reuben Matalon. Heterozygous Aspa $^{+/-}$mice in an Sv129 background were bred and newborns were genotyped on the day of birth (23,33). Briefly, 1-mm tail tips were cut and genomic DNA was extracted according to the manufacturer's protocol using either a manual QIAamp DNA Mini Kit or a QIAcube robot (Qiagen). DNA extraction was followed by quantitative PCR (qPCR). Injections were performed on P1 via the right facial vein at $4 \times 10^{11}\left(\sim 2.6 \times 10^{14}\right.$ viral genomes $/ \mathrm{kg}[\mathrm{vg} / \mathrm{kg}$; based on average $1.5 \mathrm{~g}$ weight $), 1.33 \times 10^{11}\left(\sim 8.8 \times 10^{13} \mathrm{vg} / \mathrm{kg}\right.$; based on average $1.5 \mathrm{~g}$ weight $)$, or $4 \times 10^{10}\left(\sim 2.6 \times 10^{13} \mathrm{vg} / \mathrm{kg}\right.$; based on average $1.5 \mathrm{~g}$ weight $)$ GC number of male and/or female mice. After every procedure, pups were cleaned with $70 \%$ ethanol and rubbed with bedding material. The parent animal was returned after nose numbing with $70 \%$ ethanol. 


\section{Viral production and vector design}

rAAV was produced by transient HEK 293 cell transfection and $\mathrm{CsCl}$ sedimentation as previously described (50). Vector preparations were titered by qPCR, and purity was assessed by $4 \%-12 \%$ SDS-acrylamide gel electrophoresis and silver staining (Invitrogen) as described previously (51). Morphological integrity of virions was assessed by transmission electron microscopy of negative-stained rAAV virions at the University of Massachusetts Medical School Electron Microscopy Core. Because of packaging size restrictions, we switched from selfcomplementary (sc) to single-stranded rAAV genome, which is several-fold less efficient than the sc genome for the phGFAP-hASPA and phGFAP-EGFP constructs and added an intron (52). All other vectors were sc.

\section{Western blot}

Protein was extracted using RIPA buffer. Protein quantification was performed by BCA assay (Pierce Biotechnologies) and 10-20 $\mu \mathrm{g}$ of total protein mixed with $4 \times$ Laemmli buffer (Bio-Rad) were loaded onto a $10 \%-12 \%$ Tris- $\mathrm{HCl}$ acrylamide gel (Bio-Rad). After electrophoresis, protein was blotted on a nitrocellulose membrane with the Trans-Blot Turbo Transfer System (both Bio-Rad). Subsequently, membranes were blocked at room temperature for at least 1 hour with Odyssey Blocking Buffer (Licor). Next, membranes were incubated with primary (anti-ASPA, 1:2,000, ab97454; anti-actin, 1:5,000, ab8224; both from Abcam) antibody at $4^{\circ} \mathrm{C}$ overnight and incubated with secondary antibody (Licor) the next day. The membranes were analyzed with the Odyssey analyzer (Licor). Quantification was performed using ImageJ (NIH).

\section{Isolation of brain regions and DNA and RNA extraction}

Mice were anesthetized with isoflurane and transcardially perfused with ice-cold PBS. Next, brains were removed and divided in half along the interhemispheric cleft. One brain half at a time was placed on an RNase-free and ice-cooled plate under a dissection microscope. First, the olfactory bulb was removed using a cold razor blade. Next, the brain stem/midbrain was removed along the line between the cortex/ thalamus and the lamina tecti. The brainstem was further subdivided into midbrain, lamina tecti, cerebellum, and brain stem. Furthermore, the thalamus/hypothalamus was removed using a Wecker Micro Dissecting Spatula (Roboz Surgical Instruments Inc.). The hippocampus was removed as described elsewhere (53). Finally, part of the cortex was removed with a fresh razor blade. All samples were snap frozen immediately after removal.

DNA and RNA were extracted using the Qiagen Allprep DNA/RNA Mini Kit (Qiagen). DNA was subjected to viral GC number determination.

\section{Droplet digital PCR}

Multiplex droplet digital PCR (ddPCR) was performed on a QX200 ddPCR system (Bio-Rad). All assays were based on TaqMan probes, where the gene-of-interest probes were labeled with FAM and the reference gene as VIC. Bio-Rad ddPCR mastermix with no dUTP was used (Bio-Rad, catalog 1863024) for all ddPCR reactions.

Vector GC number. DNA was digested with BamHI at greater than $10 \mathrm{U} / \mu \mathrm{g}$ of DNA at $37^{\circ} \mathrm{C}$ for 1 hour. The BamHI digest ensured single copies of rAAV genomes. All vectors contained an RBG sequence as described previously (54), which was targeted for viral genome quantification. Viral genome numbers were normalized to the number of diploid cells by using transferrin receptor (Tfrc) as the reference gene (Invitrogen, 4458367), as described previously (55).

\section{Motor function and spatial memory testing}

Mouse motor performance was assessed using accelerated rotarod for motor function and endurance, balance beam for vestibular function and ataxia, and inverted screen for grip strength. Except for the rotarod (Rotarod Series 8, IITC Life Science), the equipment was built by the University of Massachusetts Medical School Machine Shop. For each motor function test, mice $(n=8)$ were injected and tested independently.

Accelerated rotarod. Mice were trained 2 days before the testing day for 3 runs each. On the testing day, mice were placed on the rotarod to acclimate for 1 minute. Each mouse was tested 3 times and the best value was used for analysis. The acceleration and timing was set to 4 to $40 \mathrm{rpm}$ over 5 minutes.

Balance beam. To increase the stringency of this test, we increased the cut-off time from the previously used 3 minutes to 5 minutes (33). Mice were placed in the middle of the balance beam and the latency until drop off was measured. Again, the best value was counted. 
Inverted screen. Mice were placed in the center of a grid $\left(30 \mathrm{~cm}^{2}\right.$ with $25-\mathrm{mm}^{2}$ holes $)$ in horizontal position and allowed to acclimate for 1 minute. The grid was turned slowly within 15 seconds to 125 degrees so that the mouse was hanging upside down. Time was measured until drop off. The cut-off for P27 testing was 3 minutes as published previously (33). At all other time points, the cut-off was 5 minutes to increase the stringency of the test.

T maze. T-maze testing was done in a spontaneous, unrewarded manner, with all arms of the T maze open during testing. Mice were placed within the initial chamber with the door down, and the side-arm doors open for 10 seconds, upon which the initial chamber's door was opened, and the mice were allowed to enter and explore the T maze. Upon the complete entry of the mouse into one of the side arms, defined as all 4 of the paws having passed through the edge of the arm, all doors of the T maze were closed, and the mouse was returned to the initial chamber for a 10 -second resting time. During this 10 seconds, the side-arm doors were reopened. This process was repeated 10 times, which provides the mouse with a total of 10 opportunities to alternate their side-arm choice. The final result is expressed as a ratio of the number of alternations over 10 .

\section{H\&E and Luxol fast blue staining}

Mice were euthanized and perfused transcardially with ice-cold PBS and 4\% paraformaldehyde (PFA). Tissues were removed and sliced using an Alto brain or spinal cord matrix (Roboz Surgical Instruments Inc.). Subsequently, mouse tissues were stored in PFA at $4^{\circ} \mathrm{C}$ overnight. Paraffin embedding, $\mathrm{H} \& \mathrm{E}$, and Luxol fast blue staining was performed by the University of Massachusetts Medical School Morphology Core. Stained sections were analyzed and pictures taken with an Axioscope 50 (Zeiss) using a DMC2900 camera (Leica Microsystems).

\section{MRI and MRS}

Mice were anesthetized with $2 \%$ isoflurane and constantly monitored for vital signs during the entire time of imaging. P42 mice were imaged with a 4.7-T/40-cm horizontal magnet (Oxford) equipped with a Bruker Biospec/Avance console. Experiments for all other imaging were performed using the Oxford 4.7-T/40-cm horizontal magnet equipped with a Bruker Biospec/Avance III HD console. A ${ }^{1} \mathrm{H}$ radiofrequency mouse head coil (Bruker) with inner diameter of $23 \mathrm{~mm}$ was used for the experiments.

T1-weighted anatomical images were acquired using FLASH sequence with the following parameters: repetition time $(\mathrm{TR})=280.86 \mathrm{~ms}$, echo time $(\mathrm{TE})=4.5 \mathrm{~ms}$, matrix size $=384 \times 384$, field of view $($ FOV $)=18 \times 18 \mathrm{~mm}^{2}$, slice number $=15$, slice thickness $=0.5 \mathrm{~mm}$, flip angle $=40^{\circ}$, and number of averages $=8 . \mathrm{T} 2$-weighted images were acquired using TurboRARE sequence with $\mathrm{TR}=2,200 \mathrm{~ms}, \mathrm{TE}=36$ $\mathrm{ms}$, echo spacing $=12 \mathrm{~ms}, 8$ averages, and rare factor $=8 \cdot{ }^{1} \mathrm{H}$ MRS data were acquired using single-voxel point-resolved spectroscopy sequence (PRESS) (repetition time $=2,500 \mathrm{~ms}$, echo time $=16 \mathrm{~ms}$, number of averages $=512$, voxel size $=3 \times 3 \times 3 \mathrm{~mm}$ ). fMRI images were acquired for 10 minutes using echo planar imaging $(\mathrm{EPI})$ sequence, with $\mathrm{TR}=1,000 \mathrm{~ms}, \mathrm{TE}=18 \mathrm{~ms}$, matrix size $=96 \times 96, \mathrm{FOV}=18 \times 18$ $\mathrm{mm}^{2}$, slice number $=15$, slice thickness $=0.5 \mathrm{~mm}$, number of repetitions $=600$. DTI data were acquired from 30 directions with $\mathrm{B}$ value of $650 / 0, \mathrm{TR}=2,300 \mathrm{~ms}$, TE $=21 \mathrm{~ms}$, number of averages $=4$, with the same geometry parameters as EPI.

\section{${ }^{1} \mathrm{H}$ MRI and MRS study}

Proton spectra were fit using LCModel (Version 6.2-2B), which analyzed in vivo proton spectra as a linear combination of model in vitro spectra from individual metabolite solutions (56) and generated data as absolute fits (in institutional units) and $\mathrm{SD} \%$. SD was used as a measure of the reliability of the fit. The spectral inclusion criteria were SD less than $20 \%$ for NAA, creatine, and inositol.

\section{Resting state functional connectivity ( $\mathrm{rsFC}$ ) analysis}

EPI images were preprocessed using Medical Image Visualization and Analysis (MIVA, http://ccni.wpi. edu/) and Matlab 2010b (the Mathworks Inc.). All EPI images were first registered to a standard anatomy, where seed regions were defined. After registration, all EPIs went through motion correction, spatial smoothing (full width half maximum $=1 \mathrm{~mm}$ ), and $0.002-0.1 \mathrm{~Hz}$ band-pass filtering. Seed-based rsFC was calculated using a previously demonstrated algorithm (57-59). 
DTI

DTI data were analyzed using DTIstudio (https://www.mristudio.org/, Susumi Mori and Hangyi Jiang, Johns Hopkins University, Baltimore, Maryland, USA), including eddy current correction, motion correction, and generation of all tensor metrics (FA and eigen decomposition of the voxel-wise diffusion tensor). FA values in particular regions of interest (ROIs) were extracted from manually drawn ROIs.

For all imaging results, group comparisons were carried out by 1-way ANOVA, with a significance threshold of $P$ less than 0.05 .

\section{Immunohistology}

Mice were perfused transcardially with $4 \%$ PFA and kept in PFA overnight at $4{ }^{\circ} \mathrm{C}$. The next day, brains were extracted and subjected to gradient sucrose steps $(10 \%, 20 \%$, and $30 \%)$ overnight at $4^{\circ} \mathrm{C}$. Brains were mounted in OTC compound (Fisher HealthCare) and stored at $-80^{\circ} \mathrm{C}$ until cryosectioning (Cryostar NX70, Thermo Fisher Scientific). Floating brain slices were washed in $1 \times$ PBS 3 times for 5 minutes each. Cells were permeabilized with $1 \times$ PBS and $0.5 \%$ Triton-X 100 at room temperature for 1 hour, with subsequent blocking for 1 hour at room temperature with $5 \%$ serum (10\% normal goat serum, Life Technologies, 50062Z). Brain slices were incubated with primary antibodies (anti-GFAP, 1:1,000, EMD Millipore, MAB360; anti-MBP, 1:1,000, , Abcam, ab40390) in $1.5 \%$ serum overnight at $4^{\circ} \mathrm{C}$, washed the next day $(1 \times$ PBS, 3 times, 5 minutes each), and stained with secondary antibody in $1.5 \%$ serum at room temperature for 1 hour (anti-mouse or -rabbit; Invitrogen, A-11031 or A-11011). Slices were mounted using Vectashield with 4',6-diamidino-2-phenylindole (Vector Laboratories).

Brain sections were imaged and recorded using a DM 5500B upright microscope (Leica Microsystems) and Leica DFC365 FX digital camera.

\section{Software and statistics}

Image analysis and displaying was done using Imaris 8.2 Software (Bitplane Inc.). Graphs were analyzed and statistical calculations were performed in Prism 7 (GraphPad Software, Inc.). Correlation between overall functional connectivity on fMRI to mean of accelerated rotarod performance was calculated and linear regression analysis was performed using Prism 7. Statistical analyses were performed using 2-way ANOVA with multiple comparison correction (Tukey) for weights, and 1-way ANOVA with multiple comparison correction for all other statistics, if not stated otherwise. Unless stated otherwise, at least $n=3$ mice were analyzed. A $P$ value less than 0.05 was considered significant.

\section{Study approval}

All animal procedures were approved by the IACUC of the University of Massachusetts Medical School.

\section{Author contributions}

GG and DJG planned the experiments and prepared the manuscript. DJG and DL conducted the experiments and part of the animal behavioral studies. HX and ST performed part of the behavioral animal studies. QS produced the rAAV vectors. JS performed the vector titration and ddPCR analysis for vector genome copy number. CM and JK were in charge of the imaging core and analysis. RM provided the animal model and performed part of the NAA study.

\section{Acknowledgments}

We would like to thank Urmila Mallick and Jessica Epstein for their technical assistance. Funding was provided by Jacob's Cure, the National Tay-Sachs \& Allied Diseases Association, Inc., Canavan's Foundation, and NIH grants R01NS076991-01, 1P01AI100263-01, and 1P01HL131471-01.

Address correspondence to: Guangping Gao, University of Massachusetts Medical School, Horae Gene Therapy Center, 368 Plantation Street, Worcester, Massachusetts 01605, USA. Phone: 508.856.3563; E-mail: Guangping.Gao@umassmed.edu.

1. Tallan HH, Moore S, Stein WH. N-Acetyl-L-aspartic acid in brain. J Biol Chem. 1956;219(1):257-264

2. Blüml S. In vivo quantitation of cerebral metabolite concentrations using natural abundance 13C MRS at 1.5 T. JMagn Reson. 1999;136(2):219-225. 
3. Mersmann N, et al. Aspartoacylase-lacZ knockin mice: an engineered model of Canavan disease. PLoS ONE. 2011;6(5):e20336.

4. Kirmani BF, Jacobowitz DM, Namboodiri MA. Developmental increase of aspartoacylase in oligodendrocytes parallels CNS myelination. Brain Res Dev Brain Res. 2003;140(1):105-115.

5. Baslow MH, Suckow RF, Sapirstein V, Hungund BL. Expression of aspartoacylase activity in cultured rat macroglial cells is limited to oligodendrocytes. J Mol Neurosci. 1999;13(1-2):47-53.

6. Kaul R, Gao GP, Balamurugan K, Matalon R. Cloning of the human aspartoacylase cDNA and a common missense mutation in Canavan disease. Nat Genet. 1993;5(2):118-123.

7. Matalon R, Michals K, Sebesta D, Deanching M, Gashkoff P, Casanova J. Aspartoacylase deficiency and N-acetylaspartic aciduria in patients with Canavan disease. Am J Med Genet. 1988;29(2):463-471.

8. Divry P, Mathieu M. Aspartoacylase deficiency and N-acetylaspartic aciduria in patients with Canavan disease. Am JMed Genet. 1989;32(4):550-551.

9. Traeger EC, Rapin I. The clinical course of Canavan disease. Pediatr Neurol. 1998;18(3):207-212.

10. Matalon RM, Michals-Matalon K. Spongy degeneration of the brain, Canavan disease: biochemical and molecular findings Front Biosci. 2000;5:D307-D311.

11. Toft PB, et al. Magnetic resonance imaging in juvenile Canavan disease. Eur J Pediatr. 1993;152(9):750-753.

12. von Moers A, Sperner J, Michael T, Scheffner D, Schutgens RH. Variable course of Canavan disease in two boys with early infantile aspartoacylase deficiency. Dev Med Child Neurol. 1991;33(9):824-828.

13. Adachi M, Schneck L, Cara J, Volk BW. Spongy degeneration of the central nervous system (van Bogaert and Bertrand type; Canavan's disease). A review. Hum Pathol. 1973;4(3):331-347.

14. McAdams HP, Geyer CA, Done SL, Deigh D, Mitchell M, Ghaed VN. CT and MR imaging of Canavan disease. AJNR Am J Neuroradiol. 1990;11(2):397-399.

15. Beaulieu C. The basis of anisotropic water diffusion in the nervous system - a technical review. NMR Biomed. 2002;15(7-8):435455 .

16. Heeger DJ, Ress D. What does fMRI tell us about neuronal activity? Nat Rev Neurosci. 2002;3(2):142-151.

17. Baudrexel S, et al. Resting state fMRI reveals increased subthalamic nucleus-motor cortex connectivity in Parkinson's disease. Neuroimage. 2011;55(4):1728-1738.

18. Iraji A, et al. Resting state functional connectivity in mild traumatic brain injury at the acute stage: independent component and seed-based analyses. J Neurotrauma. 2015;32(14):1031-1045.

19. Johnson B, et al. Alteration of brain default network in subacute phase of injury in concussed individuals: resting-state fMRI study. Neuroimage. 2012;59(1):511-518.

20. Zhan Y, et al. Deficient neuron-microglia signaling results in impaired functional brain connectivity and social behavior. Nat Neurosci. 2014;17(3):400-406.

21. Canavan MM. Schilder's encephalitis periaxialis diffusa. Arch NeurPsych. 1931;25:299-308.

22. Leone P, et al. Long-term follow-up after gene therapy for Canavan disease. Sci Transl Med. 2012;4(165):165ra163.

23. Matalon R, et al. Knock-out mouse for Canavan disease: a model for gene transfer to the central nervous system. J Gene Med. 2000;2(3):165-175.

24. Taylor DL, et al. Investigation into the role of $\mathrm{N}$-acetylaspartate in cerebral osmoregulation. J Neurochem. 1995;65(1):275-281

25. Madhavarao CN, et al. Defective $\mathrm{N}$-acetylaspartate catabolism reduces brain acetate levels and myelin lipid synthesis in Canavan's disease. Proc Natl Acad Sci USA. 2005;102(14):5221-5226.

26. Prokesch A, et al. N-acetylaspartate catabolism determines cytosolic acetyl-CoA levels and histone acetylation in brown adipocytes. Sci Rep. 2016;6:23723.

27. Mazzoccoli C, et al. N-acetylaspartate (NAA) induces neuronal differentiation of SH-SY5Y neuroblastoma cell line and sensitizes it to chemotherapeutic agents. Oncotarget. 2016;7(18):26235-26246.

28. Madhavarao $\mathrm{CN}$, et al. Glyceryl triacetate for Canavan disease: a low-dose trial in infants and evaluation of a higher dose for toxicity in the tremor rat model. J Inherit Metab Dis. 2009;32(5):640-650.

29. Mathew R, Arun P, Madhavarao CN, Moffett JR, Namboodiri MA. Progress toward acetate supplementation therapy for Canavan disease: glyceryl triacetate administration increases acetate, but not $\mathrm{N}$-acetylaspartate, levels in brain. J Pharmacol Exp Ther. 2005;315(1):297-303.

30. Arun P, et al. Metabolic acetate therapy improves phenotype in the tremor rat model of Canavan disease. J Inherit Metab Dis 2010;33(3):195-210.

31. McPhee SW, et al. Effects of AAV-2-mediated aspartoacylase gene transfer in the tremor rat model of Canavan disease. Brain Res Mol Brain Res. 2005;135(1-2):112-121.

32. Klugmann M, Leichtlein CB, Symes CW, Serikawa T, Young D, During MJ. Restoration of aspartoacylase activity in CNS neurons does not ameliorate motor deficits and demyelination in a model of Canavan disease. Mol Ther. 2005;11(5):745-753.

33. Ahmed SS, et al. A single intravenous rAAV injection as late as P20 achieves efficacious and sustained CNS Gene therapy in Canavan mice. Mol Ther. 2013;21(12):2136-2147.

34. Cearley CN, Vandenberghe LH, Parente MK, Carnish ER, Wilson JM, Wolfe JH. Expanded repertoire of AAV vector serotypes mediate unique patterns of transduction in mouse brain. Mol Ther. 2008;16(10):1710-1718.

35. Foust KD, Nurre E, Montgomery CL, Hernandez A, Chan CM, Kaspar BK. Intravascular AAV9 preferentially targets neonatal neurons and adult astrocytes. Nat Biotechnol. 2009;27(1):59-65.

36. Gao G, et al. Adeno-associated viruses undergo substantial evolution in primates during natural infections. Proc Natl Acad Sci USA. 2003;100(10):6081-6086.

37. Gao GP, Alvira MR, Wang L, Calcedo R, Johnston J, Wilson JM. Novel adeno-associated viruses from rhesus monkeys as vectors for human gene therapy. Proc Natl Acad Sci USA. 2002;99(18):11854-11859.

38. Yang B, et al. Global CNS transduction of adult mice by intravenously delivered rAAVrh. 8 and rAAVrh.10 and nonhuman primates by rAAVrh.10. Mol Ther. 2014;22(7):1299-1309.

39. Moffett JR, Ross B, Arun P, Madhavarao CN, Namboodiri AM. N-Acetylaspartate in the CNS: from neurodiagnostics to neurobiology. Prog Neurobiol. 2007;81(2):89-131. 
40. Baslow MH, Guilfoyle DN. Are astrocytes the missing link between lack of brain aspartoacylase activity and the spongiform leukodystrophy in Canavan disease? Neurochem Res. 2009;34(9):1523-1534.

41. Maier H, Wang-Eckhardt L, Hartmann D, Gieselmann V, Eckhardt M. N-Acetylaspartate synthase deficiency corrects the myelin phenotype in a Canavan disease mouse model but does not affect survival time. J Neurosci. 2015;35(43):14501-14516.

42. Guo F, et al. Ablating N-acetylaspartate prevents leukodystrophy in a Canavan disease model. Ann Neurol. 2015;77(5):884-888.

43. Surendran S, Matalon KM, Szucs S, Tyring SK, Matalon R. Metabolic changes in the knockout mouse for Canavan's disease: implications for patients with Canavan's disease. J Child Neurol. 2003;18(9):611-615.

44. Volkenhoff A, Weiler A, Letzel M, Stehling M, Klämbt C, Schirmeier S. Glial Glycolysis is essential for neuronal survival in Drosophila. Cell Metab. 2015;22(3):437-447.

45. Casper KB, Jones K, McCarthy KD. Characterization of astrocyte-specific conditional knockouts. Genesis. 2007;45(5):292-299.

46. Furukawa-Hibi Y, et al. Absence of SHATI/Nat81 reduces social interaction in mice. Neurosci Lett. 2012;526(2):79-84.

47. Deicken RF, Pegues M, Amend D. Reduced hippocampal N-acetylaspartate without volume loss in schizophrenia. Schizophr Res. 1999;37(3):217-223.

48. Gautier EL, et al. Gata6 regulates aspartoacylase expression in resident peritoneal macrophages and controls their survival. $J$ Exp Med. 2014;211(8):1525-1531.

49. Ahmed SS, et al. rAAV gene therapy in a Canavan's disease mouse model reveals immune impairments and an extended pathology beyond the central nervous system. Mol Ther. 2016;24(6):1030-1041.

50. Sun X, Lu Y, Bish LT, Calcedo R, Wilson JM, Gao G. Molecular analysis of vector genome structures after liver transduction by conventional and self-complementary adeno-associated viral serotype vectors in murine and nonhuman primate models. Hum Gene Ther. 2010;21(6):750-761.

51. Gao GP, Sena-Esteves M. Introducing Genes into Mammalian Cells: Viral Vectors. In: Green MR, Sambrook J, eds. Molecular Cloning, Volume 2: A Laboratory Manual. New York: Cold Spring Harbor Laboratory Press;2012:1209-1313.

52. McCarty DM. Self-complementary AAV vectors; advances and applications. Mol Ther. 2008;16(10):1648-1656

53. Hagihara H, Toyama K, Yamasaki N, Miyakawa T. Dissection of hippocampal dentate gyrus from adult mouse. J Vis Exp. 2009;(33):1543.

54. Lock M, Alvira MR, Chen SJ, Wilson JM. Absolute determination of single-stranded and self-complementary adeno-associated viral vector genome titers by droplet digital PCR. Hum Gene Ther Methods. 2014;25(2):115-125.

55. Guo Y, et al. A single injection of recombinant adeno-associated virus into the lumbar cistern delivers transgene expression throughout the whole spinal cord. Mol Neurobiol. 2016;53(5):3235-3248.

56. Provencher SW. Automatic quantitation of localized in vivo 1H spectra with LCModel. NMR Biomed. 2001;14(4):260-264.

57. Liang Z, Li T, King J, Zhang N. Mapping thalamocortical networks in rat brain using resting-state functional connectivity. Neuroimage. 2013;83:237-244.

58. Liang Z, King J, Zhang N. Uncovering intrinsic connectional architecture of functional networks in awake rat brain. J Neurosci. 2011;31(10):3776-3783.

59. Zhang N, et al. Mapping resting-state brain networks in conscious animals. J Neurosci Methods. 2010;189(2):186-196. 